\title{
Telegraph Equations and Complementary Dirac Equation from Brownian Movement
}

\author{
Balwant Singh Rajput* \\ Physics Department, Kumaon University, Nainital, India \\ Email: bsrajp@gmail.com
}

Received June 15, 2012; revised August 1, 2012; accepted August 10, 2012

\begin{abstract}
Telegraph equations describing the particle densities in Brownian movement on a lattice site have been derived and it has been shown that the complementary classical Dirac equation appears naturally as the consequence of correlations in particle trajectories in Brownian movement. It has also been demonstrated that Heisenberg uncertainty relation between energy and time is the necessary and sufficient condition to transform this classical equation into usual Dirac's relativistic quantum equation.
\end{abstract}

Keywords: Telegraph Equation; Dirac Equation; Brownian Motion; Analytic Continuation; Schrödinger Equation; Uncertainty Relations

\section{Introduction}

The relativistic propagator for a free particle in $x-t$ space can be obtained [1-5] from the considerations of the statistics of random walks in space and time without restoring to formal analytic continuation. It has been shown [6] that the wave functions and propagators that occur in classical equations are themselves observables on the lattice in contrast to quantum mechanics where wave functions are rather mysterious objects which only facilitate calculations and which are not themselves observables. It has also been shown [7] that the free particle classical Schrödinger's equation in $x-t$ space occurs naturally in the description of correlations in random walks on the lattice where the wave-function solutions describe the features of ensembles of random walks. In recent attempt a stochastic model of the telegraph equation due to Kac [8] and Gaveau et al. [9] has been extended [10] to obtain Dirac Equation for a particle in electromagnetic field using Brownian motion in time as well as space. Recently, the relativistic diffusion processes have been discussed in random walk models [11] and the quantization of Brownian motion have been worked out [12].

In our earlier papers [13-15] the diffusion equation and classical Schrödinger's equation for free particle and also for a particle under a force field have been derived as complementary equations from the Brownian motion and it has been shown that the continuum limit which transforms this classical Schrödinger's equation into the usual

*Permanent address: I-11, Gamma-II, Greater Noida (UP) India.
Schrödinger's quantum equation without using any formal analytic continuation and the wave-particle duality, is simply Heisenberg's uncertainty relation between position and momentum of the Brownian particle. Extending this work in the present paper by putting a finite speed cutoff into the diffusion process we have obtained telegraphic equations to describe particle densities in Brownian movement on a lattice site and showed that the complementary classical Dirac equation appears naturally as the consequence of correlations in particle trajectories in Brownian movement. Here the constituents of wave-function describe the features of ensembles of random walks on lattice and hence the observables are easily interpreted. We have also derived the condition which transforms this classical Dirac equation for Brownian movement into usual Dirac's relativistic quantum equation and it has been demonstrated that this condition is basically Heisenberg's uncertainty relation for energy and time.

\section{Telegraphic Equations from Brownian Movement}

Let us work in 2-dimensional spatial-temporal space $(x, t)$ on a lattice with spatial and temporal spacings $\delta$ and $\varepsilon$ respectively and assume $p_{\mu}(x, t) \delta$ as the probability that a particle arrives in the state $\mu(\mu=1,2,3,4)$ at $x, t$ where these four states have been assigned to the particles to keep the track of correlation in the trajectories as they move between lattice sites such that the state1 and state- 3 correspond to the particle moving to the right and state- 2 and state- 4 correspond to the left mov- 
ing particles. The state- 1 and state- 3 are separated by an odd number of transitions from right moving to the left moving and similarly, state- 2 and state- 4 are separated by an odd number of left to right transitions. At each lattice site, the Brownian particles choose whether to go left or right at the next step. Let us assume that the particles maintain the same direction with probability $p$ and change the direction with probability $q$ such that

$$
p+q=1 \text {. }
$$

Then we write the difference equations for the ensemble of particles as

$$
\begin{aligned}
& p_{1}(x, t+\varepsilon)=p p_{1}(x-\delta, t)+q p_{4}(x+\delta, t), \\
& p_{2}(x, t+\varepsilon)=p p_{2}(x+\delta, t)+q p_{1}(x-\delta, t), \\
& p_{3}(x, t+\varepsilon)=p p_{3}(x-\delta, t)+q p_{2}(x+\delta, t), \\
& \text { and } p_{4}(x, t+\varepsilon)=p p_{4}(x+\delta, t)+q p_{3}(x-\delta, t),
\end{aligned}
$$

which is the master set of equations for the ensemble of random walks giving the distribution of particles in the four states.

Let us have

$$
Z_{1}=p_{1}+p_{2}+p_{3}+p_{4}
$$

which is proportional to the probability that a particle arrives at $(x, t)$ in any state.

Let us also define

$$
Z_{2}=p_{1}+p_{3}-p_{2}-p_{4}
$$

which gives the direction difference. If the number of left moving and right moving particles are the same in the beginning then we have

$$
Z_{2}(x, 0)=0
$$

Let $E$ be the shift operator with respect to $x$ coordinate. i.e.,

$$
\begin{aligned}
& E p_{\mu}(x, t)=p_{\mu}(x+\delta, t) \text { and } \\
& E^{-1} p_{\mu}(x, t)=p_{\mu}(x-\delta, t)
\end{aligned}
$$

Then using Equations (2.1) to (2.6), we get

$$
\begin{aligned}
& Z_{1}(x, t+\varepsilon)=1 / 2\left[E^{-1}\left(Z_{1}+Z_{2}\right)+E\left(Z_{1}-Z_{2}\right)\right] \text { and } \\
& Z_{2}(x, t+\varepsilon)=1 / 2(p-q)\left[E^{-1}\left(Z_{1}+Z_{2}\right)-E\left(Z_{1}-Z_{2}\right)\right]
\end{aligned}
$$

If we choose

$$
X_{1}=\left(Z_{1}+Z_{2}\right) / 4 \text { and } X_{2}=\left(Z_{1}-Z_{2}\right) / 4 \text {, }
$$

then Equation (2.7) reduce to

$$
\begin{aligned}
& X_{1}(x, t+\varepsilon)=p E^{-1} X_{1}(x, t)+q E X_{2}(x, t) \text { and } \\
& X_{2}(x, t+\varepsilon)=q E^{-1} X_{1}(x, t)+p E X_{2}(x, t)
\end{aligned}
$$

Expanding shift operator in terms of differential op- erator $D=\partial / \partial x$, we get

$$
\begin{aligned}
E & =1+\delta(\partial / \partial x)+\delta^{2} / 2 !\left(\partial^{2} / \partial x^{2}\right)+\delta^{3} / 3 !\left(\partial^{3} / \partial x^{3}\right) \\
& =1+\delta(\partial / \partial x)+o\left(\delta^{2}\right)
\end{aligned}
$$

Ignoring the term of order higher than $\delta$, it gives

$$
E^{ \pm n}=1 \pm n \delta(\partial / \partial x)
$$

where $n$ is any integer. Then Equation (2.7) reduce to

$$
\begin{aligned}
& Z_{1}(x, t+\varepsilon)=Z_{1}(x, t)-\delta\left(\partial Z_{2} / \partial x\right) \text { and } \\
& Z_{2}(x, t+\varepsilon)=(p-q)\left[Z_{2}(x, t)-\delta\left(\partial Z_{1} / \partial x\right)\right]
\end{aligned}
$$

If each particle persists at constant speed $c$ along its current direction for an average time $\Delta t=1 / \mu$ then

$$
\operatorname{Lim}_{\varepsilon \rightarrow 0} \delta / \varepsilon=c
$$

and

$$
q=\delta / \Delta t=\mu \varepsilon
$$

which gives

$$
p=1-q=1-\mu \varepsilon
$$

These equations show that each particle changes its direction with the average frequency $\mu$ and persists at constant speed $c$ between two consecutive changes of directions. For such a periodic motion the mean free path

$$
\lambda=c / \mu
$$

plays the role of the wave length.

Substituting the limits (2.11) and values of $p$ and $q$ from Equations (2.11a) and (2.11b) into Equation (2.10), we get

$$
\begin{aligned}
& \partial Z_{1} / \partial t=\operatorname{Lim}_{\varepsilon \rightarrow 0}\left[Z_{1}(x, t+\varepsilon)-Z_{1}(x, t)\right]=-c \partial Z_{2} / \partial x \\
& \text { and } \partial Z_{2} / \partial t=-2 \mu Z_{2}-c \partial Z_{1} / \partial x+2 \mu \delta \partial Z_{1} / \partial x
\end{aligned}
$$

Then Equations (2.8) reduce to the following form

$$
\begin{aligned}
& \partial X_{1} / \partial t=-c \partial X_{1} / \partial x-\mu\left(X_{1}-X_{2}\right)+\mu \delta \partial / \partial x\left(X_{1}+X_{2}\right) \\
& \text { and } \\
& \partial X_{2} / \partial t=c \partial X_{2} / \partial x+\mu\left(X_{1}-X_{2}\right)-\mu \delta \partial / \partial x\left(X_{1}+X_{2}\right)
\end{aligned}
$$

where $\mu \delta=\delta^{2} / \varepsilon \lambda$ and it may be ignored if we retain the terms of only first order in $\delta$ and then Equations (2.13) and (2.14) respectively reduce to the following forms:

$$
\begin{aligned}
& \partial Z_{1} / \partial t=-c \partial Z_{2} / \partial x, \\
& \partial Z_{2} / \partial t=-2 \mu Z_{2}-c \partial Z_{1} / \partial x \text { and } \\
& \partial X_{1} / \partial t=-c \partial X_{1} / \partial x-\mu\left(X_{1}-X_{2}\right), \\
& \partial X_{2} / \partial t=c \partial X_{2} / \partial t+\mu\left(X_{1}-X_{2}\right) .
\end{aligned}
$$

where Equations (2.16) give a particular form of Telegraph equations. 
Imposing the condition (2.5), Equations (2.15) are further reduced to

$$
\begin{aligned}
& \partial Z_{1} / \partial t=-c \partial Z_{2} / \partial x, \text { and } \\
& \partial Z_{2} / \partial t=-c \partial Z_{1} / \partial x
\end{aligned}
$$

which give another form of Telegraph equations.

\section{Classical Dirac Equation}

Let us denote the expected excess in the number of Brownian particles moving in a given direction by parity,

$$
\phi_{1}=p_{1}-p_{3} \text { and } \phi_{2}=p_{2}-p_{4}
$$

which correspond to the expected difference in the number of even and odd parity paths to a given point. Then using Equation (2.2) we have

$$
\begin{aligned}
& \phi_{1}(x, t+\varepsilon)=p E^{-1} \phi_{1}-q E \phi_{2} \text { and } \\
& \phi_{2}(x, t+\varepsilon)=q E^{-1} \phi_{1}+p E \phi_{2}
\end{aligned}
$$

The ensemble of particles, described by master Equations (2.2), change its state with each step and it takes eight time steps for the ensemble to return to its initial statistical state in the sense that the expected number of direction changes per particle is four [6]. Thus the Equation (3.2) in the continuum limit is iterated for eight time steps i.e.

$$
\partial \phi_{k} / \partial t=\operatorname{Lim}_{\varepsilon \rightarrow 0}\left[\phi_{k}(x, t+8 \varepsilon)-\phi_{k}(x, t)\right] / 8 \varepsilon
$$

where $k=1,2$, and

$$
\left(\begin{array}{l}
\phi_{1}(x, t+8 \varepsilon) \\
\phi_{2}(x, t+8 \varepsilon)
\end{array}\right)=\hat{A}^{8}\left(\begin{array}{l}
\phi_{1}(x, t) \\
\phi_{2}(x, t)
\end{array}\right)
$$

with

$$
\hat{A}=\left(\begin{array}{c}
p E^{-1}-q E \\
q E^{-1} p E
\end{array}\right)
$$

or

$$
\hat{A}^{8}=\left(\begin{array}{cc}
p^{8}(1-8 \delta \partial / \partial x) & -8 p^{7} q(1+\delta \partial / \partial x) \\
8 p^{7} q(1-\delta \partial / \partial x) & p^{8}(1+8 \delta \partial / \partial x)
\end{array}\right)
$$

under the approximation (2.9). Here under the first order approximation various powers of $p$, given by (2.11b) may be written as

$$
p^{n}=1-n \varepsilon \mu
$$

and then Equation (3.5) becomes

$$
\hat{A}^{8}=\left(\begin{array}{cc}
1-8 \mu \varepsilon-8 \delta \partial / \partial x & -8 \varepsilon \mu \\
8 \varepsilon \mu & 1-8 \mu \varepsilon+8 \delta \partial / \partial x
\end{array}\right)
$$

Substituting this result into Equation (3.4) and using Equation (3.3), we get

$$
\begin{aligned}
& \partial \phi_{1} / \partial t=-\mu\left(\phi_{1}+\phi_{2}\right)-c \partial \phi_{1} / \partial x \text { and } \\
& \partial \phi_{2} / \partial t=\mu\left(\phi_{1}-\phi_{2}\right)+c \partial \phi_{2} / \partial x
\end{aligned}
$$

Let us write

$$
\begin{aligned}
& \psi_{2}(x, t)=\phi_{1}(x, t) e^{\mu t} \text { and } \\
& \psi_{3}(x, t)=\phi_{2}(x, t) e^{\mu t}
\end{aligned}
$$

Then Equation (3.7) become

$$
\begin{aligned}
& \partial \psi_{2} / \partial t=-c \partial \psi_{2} / \partial x-\mu \psi_{3} \text { and } \\
& \partial \psi_{3} / \partial t=c \partial \psi_{3} / \partial x+\mu \psi_{2}
\end{aligned}
$$

Let us revert the Brownian motion such that the statesone and three correspond to particles moving to the left while the states-two and four correspond to right moving particles. In this case the states-one and three will be separated by an odd number of transitions from left moving to right moving and the states-two and four will be separated by an odd number of right to left transitions. Then the master Equations (2.2) will be transformed to

$$
\begin{aligned}
& p_{1}(x, t+\varepsilon)=p p_{1}(x+\delta, t)+q p_{4}(x-\delta, t) \\
& p_{2}(x, t+\varepsilon)=p p_{2}(x-\delta, t)+q p_{1}(x+\delta, t) \\
& p_{3}(x, t+\varepsilon)=p p_{3}(x+\delta, t)+q p_{2}(x-\delta, t) \\
& p_{4}(x, t+\varepsilon)=p p_{4}(x-\delta, t)+q p_{3}(x+\delta, t)
\end{aligned}
$$

If $\phi_{3}=p_{1}-p_{3}$ and $\phi_{3}=p_{2}-p_{4}$ are the expected excesses in the number of left moving and right moving particles respectively, then we have

$$
\begin{aligned}
& \Phi_{3}(x, t+\varepsilon)=p E \phi_{3}(x, t)-q E^{-1} \phi_{4} \text { and } \\
& \Phi_{4}(x, t+\varepsilon)=p E^{-1} \phi_{4}(x, t)+q E \phi_{3}
\end{aligned}
$$

Then Equation (3.4) becomes

$$
\left(\begin{array}{l}
\Phi_{3}(x, t+\varepsilon) \\
\Phi_{4}(x, t+\varepsilon)
\end{array}\right)=B^{8}\left(\begin{array}{l}
\phi_{3}(x, t) \\
\phi_{4}(x, t)
\end{array}\right)
$$

where

$$
B=\left(\begin{array}{cc}
p E & -q E^{-1} \\
q E & p E^{-1}
\end{array}\right)
$$

Thus in place of Equations (3.7), we have

$$
\begin{aligned}
& \partial \Phi_{3} / \partial t=c \partial \Phi_{3} / \partial x-\mu\left(\Phi_{3}+\Phi_{4}\right) \text { and } \\
& \partial \Phi_{4} / \partial t=-c \partial \Phi_{4} / \partial x+\mu\left(\Phi_{3}-\Phi_{4}\right)
\end{aligned}
$$

Let us choose

$$
\begin{aligned}
& \psi_{1}(x, t)=\Phi_{3}(x, t) e^{\mu t} \text { and } \\
& \psi_{4}(x, t)=\Phi_{4}(x, t) e^{\mu t}
\end{aligned}
$$

Then Equation (3.14) reduce to

$$
\begin{aligned}
& \partial \psi_{1} / \partial t=c \partial \psi_{1} / \partial x-\mu \psi_{4} \text { and } \\
& \partial \psi_{4} / \partial t=-c \partial \psi_{4} / \partial x+\mu \psi_{1}
\end{aligned}
$$


If we define the function

$$
\psi=\left[\begin{array}{l}
\psi_{1} \\
\psi_{2} \\
\psi_{3} \\
\psi_{4}
\end{array}\right]
$$

then Equations (3.9) and (3.15) may be written as

$$
\gamma_{1} \partial \psi / \partial x+\gamma_{4 / i c} \partial \psi / \partial x+K \psi=0
$$

where $K=\mu / c$ and $\gamma_{1}$ and $\gamma_{4}$ are $4 \times 4$ matrices defined as

$$
\gamma_{1}=\left(\begin{array}{cccc}
0 & 0 & 0 & -1 \\
0 & 0 & 1 & 0 \\
0 & 1 & 0 & 0 \\
-1 & 0 & 0 & 0
\end{array}\right), \gamma_{4}=\left(\begin{array}{cccc}
0 & 0 & 0 & -1 \\
0 & 0 & -1 & 0 \\
0 & 1 & 0 & 0 \\
1 & 0 & 0 & 0
\end{array}\right)
$$

satisfying the conditions

$$
\gamma_{1}^{2}=\gamma_{4}^{2}=I, \gamma_{1} \gamma_{4}+\gamma_{4} \gamma_{1}=0
$$

These equations may be generalized in to the following form in three dimensional spatial coordinates;

$$
\left(\gamma_{\mu} \partial_{\mu}+K\right) \psi=0
$$

where $\mu=1,2,3,4$ with $\partial_{\mu}=\partial / \partial x_{\mu}$ and $\gamma_{2}$ and $\gamma_{3}$ are given by

$$
\gamma_{2}=\left(\begin{array}{cccc}
1 & 0 & 0 & 0 \\
0 & 1 & 0 & 0 \\
0 & 0 & -1 & 0 \\
0 & 0 & 0 & -1
\end{array}\right), \gamma_{3}=\left(\begin{array}{cccc}
0 & 0 & -1 & 0 \\
0 & 0 & 0 & -1 \\
-1 & 0 & 0 & 0 \\
0 & -1 & 0 & 0
\end{array}\right)
$$

which satisfy the following conditions

$$
\gamma_{2}^{2}=\gamma_{3}^{2}=1 \text { and } \gamma_{2} \gamma_{3}+\gamma_{3} \gamma_{2}=I
$$

These matrices also satisfy the following conditions with matrices $\gamma_{1}$ and $\gamma_{4}$ given by Equation (3.17);

$$
\begin{aligned}
& \gamma_{1} \gamma_{2}+\gamma_{2} \gamma_{1}=0 ; \gamma_{1} \gamma_{3}+\gamma_{3} \gamma_{1}=0 ; \gamma_{2} \gamma_{4}+\gamma_{4} \gamma_{2}=0 \\
& \text { and } \gamma_{3} \gamma_{4}+\gamma_{4} \gamma_{3}=0 .
\end{aligned}
$$

The conditions (3.18), (3.18a) and (3.18b) may be combined into following form

$$
\gamma_{\mu} \gamma_{v}+\gamma_{v} \gamma_{\mu}=2 \delta_{\mu v} .
$$

These matrices constitute Majorana representation of Dirac operators and hence Equation (3.19) may be called classical Dirac equation which becomes usual relativistic Dirac equation for

$$
K=\mu / c=m o c / h,
$$

where $c$ is the velocity of light and $\hbar=h / 2 \pi$ with $h$ as Planck's constant

This condition may be interpreted as

$$
\hbar=m o c^{2} / \mu
$$

But we have already seen that $\mu=1 / \Delta t$ where $\Delta t$ is the average time for which each particle in the Brownian motion persists at constant speed $c$ along its current direction. $m o c^{2}$ is its rest energy during $\Delta t$ and thereafter it changes the direction. In other words in the time interval $\Delta t$ the change of energy is $\Delta E=m o c^{2}$. Then the condition (3.22), under which the classical Dirac equation becomes the Dirac's relativistic quantum equation, is

$$
\Delta E \cdot \Delta t=\hbar
$$

which is Heisenberg's uncertainty relation for energy and time.

\section{Discussion}

Equations (2.16) and (2.17), obtained from the Brownian movement represented by master Equations (2.2), give two different forms of Telegraph equation. The classical Dirac Equation (3.16) in one spatial dimension and its generalization into the form given by Equation (3.19) appear naturally as the consequence of master Equations (2.2) and (3.10) of Brownian movement, where the constituents of $\psi$, given by Equation (3.15a), have the meaning on the lattice and denote the limits of ensemble averages of excess parities. These functions are not just the calculational tools and the function $\psi$ describes the features of ensembles of random walks on lattice and hence the observables are easily interpreted in contrast to the usual quantum mechanics.

The conditions (3.22) which transforms the classical Dirac equation for Brownian movement into usual Dirac's relativistic quantum equation, is basically Heisenberg's uncertainly relation (3.23) for energy and time. The usual formal analytic continuation which is necessary to relate the classical and quantum equation is completely absent here and hence the interpretation of quantum mechanics here is direct one without the problems of measurements usually associated with quantum mechanics. Here the derivation of Dirac equation is a sensible classical scheme to produce many particle simulations of quantum mechanics where quantum equation exists as a description of classical theory (Brownian movement).

In our earlier papers [13-15] it has been shown that for transforming the classical Schrödinger's equations, obtained as the consequence of Brownian movement, into usual Schrödinger's quantum equation the necessary condition is Heisenberg's uncertainty relation between position and momentum of the Brownian particle. In the light of this result and the forgoing discussion it may be concluded that the classical equations for ensemble averages of excess parity in Brownian movement can be transformed into usual Schrödinger's equation by imposing Heisenberg's uncertainty relation between posi- 
tion and momentum of Brownian particle and the similar classical equations can be transformed into usual Dirac's equation by imposing the uncertainty relation between energy and time associated with Brownian particle without using a formal analytic continuation and wave-particle quality. These results support the recent work [16] on the role of generalized uncertainty principle in the development of quantum mechanics from classical context. These results partially support the earlier work [1-5] showing that the quantum mechanical equations are the derived properties of the binomial distribution and no formal analytic continuation is required to produce them. Some results of this paper shall be helpful in framing the foundation of space-time path formalism [17] for relativistic quantum mechanics. We shall undertake the study of this problem in our forthcoming paper.

\section{REFERENCES}

[1] G. N. Ord, "Classical Analog of Quantum Phase," International Journal of Theoretical Physics, Vol. 31, No. 7, 1992, pp. 1177-1195. doi:10.1007/BF00673919

[2] D. G. C. McKeon and G. N. Ord, "Time Reversal in Stochastic Processes and the Dirac Equation," Physical Review Letters, Vol. 69, No. 1, 1992, pp. 3-4.

[3] G. N. Ord, "A Reformulation of the Feynman Chessboard Model," Journal of Statistical Physics, Vol. 66, No. 1-2, 1992, pp. 647-659. doi:10.1007/BF01060086

[4] G. N. Ord, "Quantum Interference from Charge Conservation," Physics Letters A, Vol. 173, No. 4-5, 1993, pp. 343-346. doi:10.1016/0375-9601(93)90247-W

[5] G. N. Ord, "Schrödinger's Equation and Discrete Random Walks in a Potential Field," Annals of Physics, Vol. 250, No. 1, 1996, pp. 63-68. doi:10.1006/aphy.1996.0088

[6] G. N. Ord, "The Schrödinger and Dirac Free Particle Equations without Quantum Mechanics," Annals of Physics, Vol. 250. No. 1, 1996, pp. 51-62. doi:10.1006/aphy.1996.0087

[7] G. N. Ord and A. S. Deakin, "Random Walks, Continuum
Limits, and Schrödinger's Equation," Physical Review A, Vol. 54, No. 5, 1996, pp. 3772-3778. doi:10.1103/PhysRevA.54.3772

[8] M. Kac and Mt. Rocky, "A Stochastic Model Related to the Telegrapher's Equation," Rocky Mountain Journal of Mathematics, Vol. 4, No. 3, 1974, pp. 494-510.

[9] M. Kac, B. Gaveau, T. Jacobson and L. Schulman, "Relativistic Extension of the Analogy between Quantum Mechanics and Brownian Motion," Physical Review Letters, 53, No. 5, 1984, pp. 419-422.

[10] D. G. C. McKeon and G. N. Ord, "Time Reversal and a Stochastic Model of the Dirac Equation in an Electromagnetic Field," Canadian Journal of Physics, Vol. 82, No. 1, 2004, pp. 19-27.

[11] J. Dunkel, P. Talkner and P. Hanggi, "Relativistic Diffusion Processes and Random Walk Models," Physical Review D, Vol. 75, No. 4, 2007, Article ID: 043001. doi:10.1103/PhysRevD.75.043001

[12] E. M. Rabei, A.-W. Ajlouni and B. Humam, "Quantization of Brownian Motion," International Journal of Theoretical Physics, Vol. 45, No. 9, 2006, 1613-1623. doi:10.1007/s10773-005-9001-3

[13] B. S. Rajput, "Telegraph Equations and Complementary Dirac Equation from Classical Approach," Acta Ciencia Indica, Vol. 36, No. 1, 2010, pp. 81-88.

[14] B. S. Rajput, "Quantum Equations from Brownian Motion," Canadian Journal of Physics, Vol. 89, No. 2, 2011, pp. 185-191. doi:10.1139/P10-111

[15] B. S. Rajput, "Quantum Equations from Classical Approach," Indian Journal of Physics, Vol. 85, No. 12, 2010, pp. 1817-1828. doi:10.1007/s12648-011-0195-3

[16] J. Y. Bang and M. S. Berger, "Possible Equilibria of Interacting Dark Energy Models," Physical Review D, Vol. 74, No. 12, 2006, Article ID: 125012. doi:10.1103/PhysRevD.74.125012

[17] Ed. Seidewitz, "Foundations of a Spacetime Path Formalism for Relativistic Quantum Mechanics," Journal of Mathematical Physics, Vol. 47, No. 11, 2006, Article ID: 112302. 